\title{
Photoelectrocatalytic Degradation of Sulfosalicylic Acid and Its Electrochemical Impedance Spectroscopy Investigation
}

\author{
Hong Liu, ${ }^{*}{ }^{\dagger}$ Shaoan Cheng, ${ }^{\ddagger}$ Ming Wu, ${ }^{\dagger}$ Hejin Wu,${ }^{\dagger}$ Jianqing Zhang, ${ }^{\ddagger}$ Wenzhao Li, ${ }^{\dagger}$ and \\ Chunan Cao \\ State Key Laboratory of Catalysis, Dalian Institute of Physical Chemistry, Chinese Academy of Sciences, \\ Dalian 116023, China, and Department of Chemistry, Zhejiang University, Hangzhou 310027, China
}

Received: January 12, 2000; In Final Form: March 27, 2000

\begin{abstract}
A three-electrode system composed of $\mathrm{TiO}_{2} / \mathrm{Ni}$ as the working electrode, porous nickel as the counter electrode, and saturated calomel electrode (SCE) as the reference electrode was used for the photoelectrocatalytic degradation of organic compounds. The photoelectrocatalytic degradation of sulfosalicylic acid (SSal) under anodic bias potential was investigated. It is shown that SSal can be degraded effectively as the external potential is increased up to $700 \mathrm{mV}$ (vs SCE). The characteristics by electrochemical impedance spectroscopy (EIS) of the photoelectrocatalytic degradation of sulfosalicylic acid (SSal) was also investigated. It is shown from the EIS that the photoelectrocatalytic degradation appears to be a simple reaction on the electrode surface, suggesting that only one step of charge transfer is involved in the electrode process. The value of the resistance of charge transfer for the photoelectrocatalytic reaction of SSal manifests itself not only in the reaction rate, but also in the separation efficiency of the photogenerated electron-hole pairs. The separation efficiency of the electron-hole pairs under $\mathrm{N}_{2}$ atmosphere is higher than that under $\mathrm{O}_{2}$ atmosphere.
\end{abstract}

\section{Introduction}

Photocatalysis of the $\mathrm{TiO}_{2}$ semiconductor has received increasing attention for the application of degrading a great variety of organic contaminants in water. ${ }^{1-4}$ The interest lies in the simplicity and low cost of the photocatalytic system which is mainly composed of an ultraviolet or a visible light source, the $\mathrm{TiO}_{2}$, and atmospheric $\mathrm{O}_{2}$. Accordingly, commercial interest is increasing. However, two problems must be overcome before the $\mathrm{TiO}_{2}$ photocatalytic system can be put into practical use. One is to reuse the photocatalyst, and the other is to decrease the simple recombination efficiency of the photogenerated electrons and holes. Some kinds of fixation techniques ${ }^{5}$ have been adopted to prevent the separation process from reusing the photocatalyst. As far as the second problem is concerned, an externally applied anodic bias is proposed to improve the charge separation by driving the photogenerated electrons via the external circuit to the counter electrode, and the degradation of several organic compounds has been reported to be effective. ${ }^{6-10}$ In fact, the pioneering work of photocatalysis was conducted in an electrode system by Fujishima and Honda. ${ }^{11}$

Electrochemical methods have also been employed in direct wastewater treatments, for example, for the decoloration and degradation of recalcitrant pollutants such as cyanide, EDTA, and aniline. ${ }^{13}$ Rajeshwar has made an interesting review about electrochemistry and the environment. ${ }^{12}$ On the other hand, electrochemical measurements are found to be useful in the direct observation of $\mathrm{TiO}_{2}$ surface reactions on the electrode. Some researchers have confirmed the synergic effect between the electrochemical and photoelectrochemical processes used to degrade reactive dyes by voltammetric results. ${ }^{14}$ Kesselman et al. stated that no individual process, i.e., electron transfer,

* Author to whom correspondence should be addressed. Fax: 86-4114691570. E-mail: hongliu203@ hotmail.com.

Dalian Institute of Physical Chemistry, Chinese Academy of Sciences.

$\doteqdot$ Department of Chemistry, Zhejiang University. hole transfer or the recombination of electrons and holes, was "rate-determining" by flux-matching conditions applied to $\mathrm{TiO}_{2}$ photoelecrodes. ${ }^{15}$

As a widely used electrochemical method, electrochemical impedance spectroscopy (EIS) is very effective for studying the mechanism and kinetics of complicated electrode reaction. Gomes and Vanmaekelbergh reviewed the impedance spectroscopy at semiconductor electrodes. ${ }^{16}$ Hens highlighted the electrochemical impedance of the electrode process involving charge transfer. ${ }^{17}$ Actually, the photoelectrochemical or electrochemically assisted photocatalytic degradation reactions of organic compounds ${ }^{6-10,14}$ can be considered as electrode reactions taking place on the electrode/liquid interface. The function of an anodic bias is to increase the separation efficiency of photogenerated electron-hole $(\mathrm{e}-\mathrm{h})$ pairs and to act as the electrode potential in the kinetic process. Therefore, the EIS method might provide some useful information if it is employed to investigate photoelectrochemical degradation reactions. In this paper, the photoelectrocatalytic degradation of sulfosalicylic acid (SSal) and its EIS investigation is reported.

\section{Experimental Section}

$\mathrm{TiO}_{2}$ (anatase) was laboratory reagent grade, and was obtained from Shanghai Chemical Factory. The average radius of the particle is $458 \mathrm{~nm}$. Other chemicals were analytical reagent grade, and SSal was purified by recrystallization before it was used for the EIS measurement. Nitrogen was highly pure and oxygen was generally pure. The solution $\mathrm{pH}$ values were adjusted by $0.01 \mathrm{~mol} \mathrm{~L}^{-1} \mathrm{HClO}_{4}$ and $0.01 \mathrm{~mol} \mathrm{~L}^{-1} \mathrm{NaOH}$ solutions before the reactions. Distilled water was used throughout this work. Porous nickel (porosity $>95 \%$ ) was washed with a dilute alkaline solution $\left(\mathrm{NaOH}: \mathrm{Na}_{2} \mathrm{CO}_{3}=1: 1\right)$ prior to use. The $\mathrm{TiO}_{2}$ powder was packed into the porous nickel substrate using $3 \mathrm{wt} \%$ poly(vinyl alcohol) (PVA) as the binder. The thickness of the immobilized $\mathrm{TiO}_{2}$ was $0.6 \mathrm{~mm}$. Figure 1 gives 


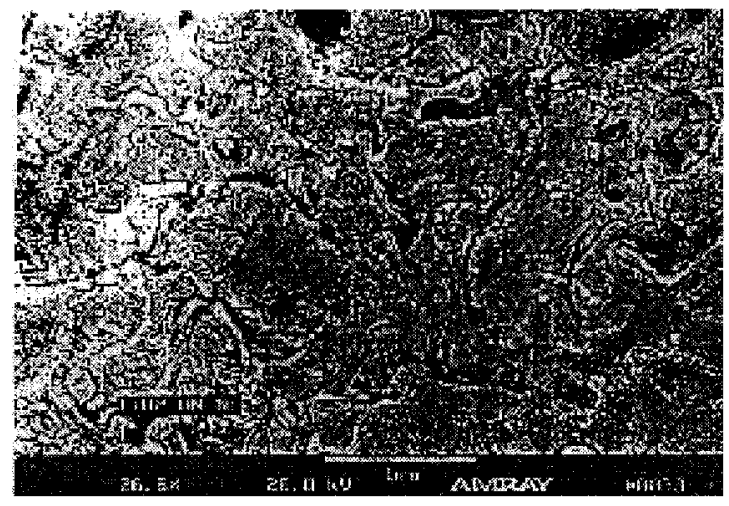

Figure 1. The SEM picture of $\mathrm{TiO}_{2} / \mathrm{Ni}$.

its morphology. The used catalyst was washed, dried by heating, and reused. All the serial experiments were performed on the same immobilized $\mathrm{TiO}_{2}$, and the reproducibility of the parallel experiments was tested to be satisfactory.

SSal can form the metal complex of $[\mathrm{Fe}(\mathrm{SSal})]^{3+}$ with ferric ion when the solution $\mathrm{pH}$ value is smaller than 2.5 . This complex exhibits characteristic absorbance at $500 \mathrm{~nm}$. Therefore, the concentration of SSal was determined by the addition of a certain amount of excessive ferric ion. The sample solution for analysis was acidified by $0.01 \mathrm{~mol} \mathrm{~L}^{-1} \mathrm{HClO}_{4}$ solution. A Shanghai 752 UV-vis spectrosphotometer was employed to measure the absorbance of $[\mathrm{Fe}(\mathrm{SSal})]^{3+}$ to calculate the concentration of the SSal.

The photoelectrochemical degradation reactions were performed in a three- electrode system in which $\mathrm{TiO}_{2} / \mathrm{Ni}$ acted as the working electrode (area $120 \mathrm{~cm}^{2}$ ), porous nickel as the counter electrode (area $>120 \mathrm{~cm}^{2}$ ), and saturated calomel electrode (SCE) as the reference electrode. The volume of the reactor was $150 \mathrm{~mL}$. External bias potential was applied by a potentiostat (ZF-5, Shanghai). A $6 \mathrm{~W}, 365 \mathrm{~nm}$ lamp was used as the light source. To eliminate the effect of adsorption on the analytical procedure, the lamp was not turned out until adsorption equilibrium was established according to the results of adsorption kinetics (see ref 18). The temperature was held at $22 \pm 1{ }^{\circ} \mathrm{C}$ by a thermostat.

The electrochemical measurements were performed with an SI 1287 potentiostat/galvanostat and a 1250 frequency response analyzer (Solartron). The three-electrode system was also composed of $\mathrm{TiO}_{2} / \mathrm{Ni}$ as the working electrode $\left(4 \mathrm{~cm}^{2}\right)$, porous nickel as the counter electrode $\left(6 \mathrm{~cm}^{2}\right)$, and a saturated calomel electrode (SCE) as the reference electrode, while the ratio between the solid and liquid in the measurement system is much larger than that in the photoelectrochemical degradation system. The impedance data were collected as a function of frequency scanned from the highest $\left(10^{5} \mathrm{~Hz}\right)$ to the lowest $(0.005 \mathrm{~Hz})$. The data of photo current response were collected at open potential. A $6 \mathrm{~W} 365 \mathrm{~nm}$ lamp was employed if needed.

\section{Results and Discussion}

Photoelectrocatalytic Degradation of SSal. No considerable decrease of SSal concentration was observed in the absence of external anodic bias or illumination, i.e., the degradation rates were not up to $10 \%$ after $120 \mathrm{~min}$. Only in the presence of external anodic bias and illumination could obvious SSal concentration decrease be detected. The experimental results are given in Table 1 and Figure 2, in which it is shown that a $0.7 \mathrm{~V}$ potential is enough to photoelectrochemically degrade SSal under our conditions.

It is hard to ascertain the degradation products by GC-MS because the SSal is a kind of organic acid. But we observed by
TABLE 1: External Bias and First-Order Reaction Constants $^{a}$

\begin{tabular}{cl}
\hline $\begin{array}{c}\text { external potential } \\
(\mathrm{mV})\end{array}$ & $\begin{array}{c}\text { first-order constant } \\
\left(\mathrm{min}^{-1}\right)\end{array}$ \\
\hline 500 & $0.0014(r=0.966)$ \\
600 & $0.00349(r=0.987)$ \\
700 & $0.0153(r=0.997)$ \\
800 & $0.0148(r=0.965)$
\end{tabular}

${ }^{a} C_{0}=1.91 \times 10^{-4} \mathrm{~mol} \mathrm{~L}^{-1}$, reaction $120 \mathrm{~min}, \mathrm{~N}_{2}$ flow $=1000 \mathrm{~mL}$ $\min ^{-1}, \mathrm{pH}=7.5$.

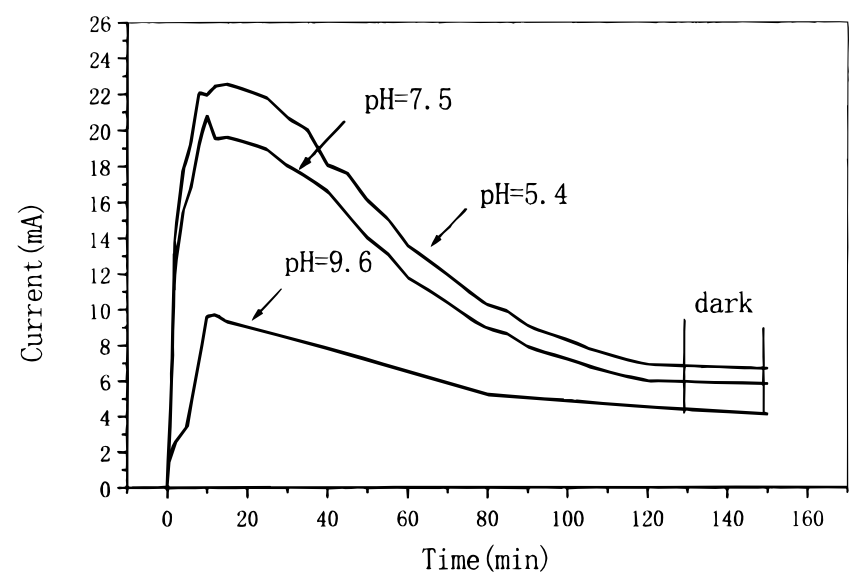

Figure 2. Current change in the course of photoelectrocatalytic degradation: bias $=700 \mathrm{mV}$, SSal concentration $=1.97 \times 10^{-4} \mathrm{~mol}$

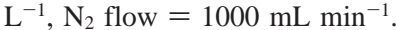

HPLC that the peaks of the degradation intermediates and the products disappeared within $180 \mathrm{~min}$. Besides, no effect of the existence of the intermediates on the analytical results of the SSal concentration was found.

Current Change in Photoelectrocatalytic Degradation. During the course of photoelectrochemical degradation with an external anodic potential, the current characteristics in the external circuit are shown in Figure 2. The current values decreased with time, indicating that the current change was dependent on the resistance of the surface reaction. When the illumination was stopped, the current change with time was very slow.

pH Effect on the Degradation Reaction. Solution pH is an important factor affecting the kinetics of the photoelectrocatalytic degradation of organic compounds because the $\mathrm{pH}$ influences the adsorption/desorption of the organics on $\mathrm{TiO}_{2}$. And it is found that the larger the amount of the organics adsorbed on $\mathrm{TiO}_{2}$, the more rapidly degradation occurs. ${ }^{18,19}$ However, for the photoelectrocatalytic degradation when a bias is involved, the case is not the same. In the present work, the $\mathrm{pH}$ dependence of the reaction rate follows the order $\mathrm{pH} 5.4 \approx \mathrm{pH} 7.5>\mathrm{pH}$ 9.6 (Figure 3), which reflects the order followed by the dependence of current on solution $\mathrm{pH}$ (see Figure 2), not the order of the adsorption ability of the $\mathrm{SSal}$ on the $\mathrm{TiO}_{2} / \mathrm{Ni}$ (see ref 18). These results indicate the direct correlation between the degradation reaction rate and the current value of the external circuit. Hidaka et al. ${ }^{20}$ also found that under an applied bias, the larger evolution of photocurrent in the photoelectrochemical degradation of several amino acids corresponded to a more rapid degradation. Therefore, it could be concluded that the solution $\mathrm{pH}$ influences the carrier flux passing through the external circuit, thus affecting the reaction rates of the SSal degradation.

Effect of Oxygen. Oxygen is essential in the photocatalytic degradation without external anodic bias, thus air or oxygen must be supplied. The photoelectrochemical degradation system, 


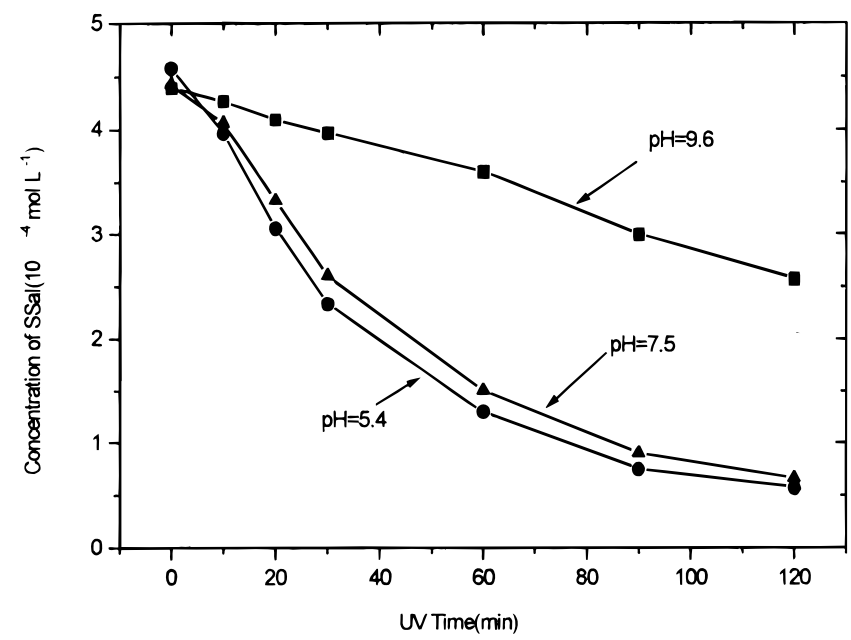

Figure 3. Photoelectrocatalytic degradation of SSal under different $\mathrm{pH}$ values: bias $=700 \mathrm{mV}, \mathrm{N}_{2}$ flow $=1000 \mathrm{~mL} \mathrm{~min}{ }^{-1}$.

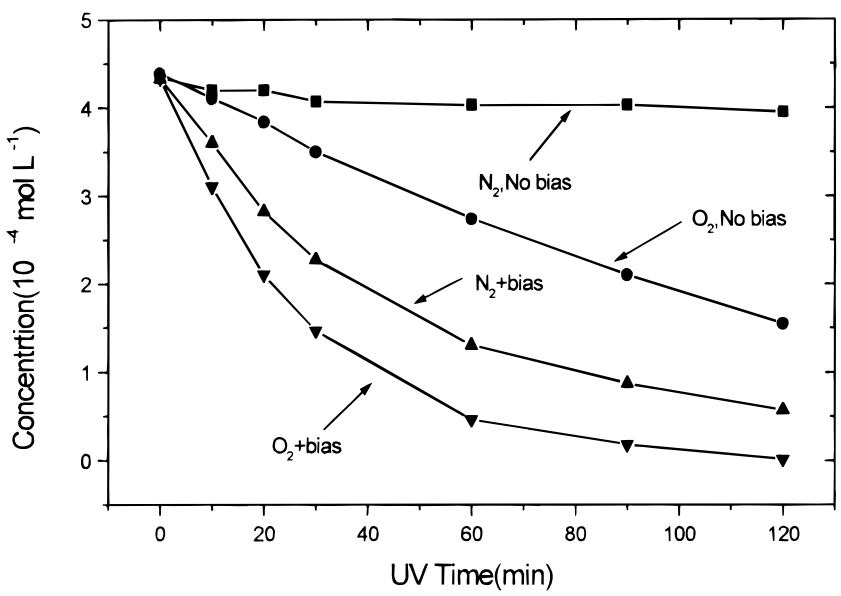

Figure 4. Comparison of photoelectrochemical degradation in $\mathrm{O}_{2}$ - and $\mathrm{N}_{2}$-purged solutions: bias $=0.7 \mathrm{~V}$ (vs SCE), gas flow $=1000 \mathrm{~mL}$ $\min ^{-1}$.

however, can be used in anaerobic conditions. ${ }^{7}$ Vinodgopal et al. ${ }^{21}$ attribute the indirect influence of $\mathrm{O}_{2}$ on the electrochemically assisted photocatalytic reaction mechanism to two aspects: (i) influence on the surface chemical process, and (ii) enhanced degradation of reaction intermediates, whereas reduced or activated oxygen species do not seem to influence the reaction course significantly under their experimental conditions.

In the present work, $\mathrm{N}_{2}$ was purged into the solutions and the reaction system was posed in a single pool; therefore, the amount of $\mathrm{O}_{2}$ could be neglected. This kind of concept resembles that of Kim and Anderson, ${ }^{7}$ and part of Vinodgopal et al. ${ }^{21}$ Figure 4 compares results of photoelectrochemical degradations under different conditions. It is shown that the degradation rate under $\mathrm{O}_{2}$-purged and external bias condition is larger, but not much, than that under $\mathrm{N}_{2}$-purged and external bias condition. $\mathrm{O}_{2}$ is not necessary for the photoelectrochemical degradation of SSal to proceed under our experimental conditions. The results are in agreement with those obtained in ref 7 . Moreover, the following phenomena were observed in this work: the solution $\mathrm{pH}$ value in the presence of $\mathrm{N}_{2}$ during the degradation changed more drastically from neutral to basic than that in the presence of $\mathrm{O}_{2}$ (Figure 5), and the external current change was also considerably larger under the $\mathrm{N}_{2}$-purged condition (see Figure 6).

On the basis of the above results, it seems reasonable that $\mathrm{H}^{+}$is involved in the degradation and acts as the electron

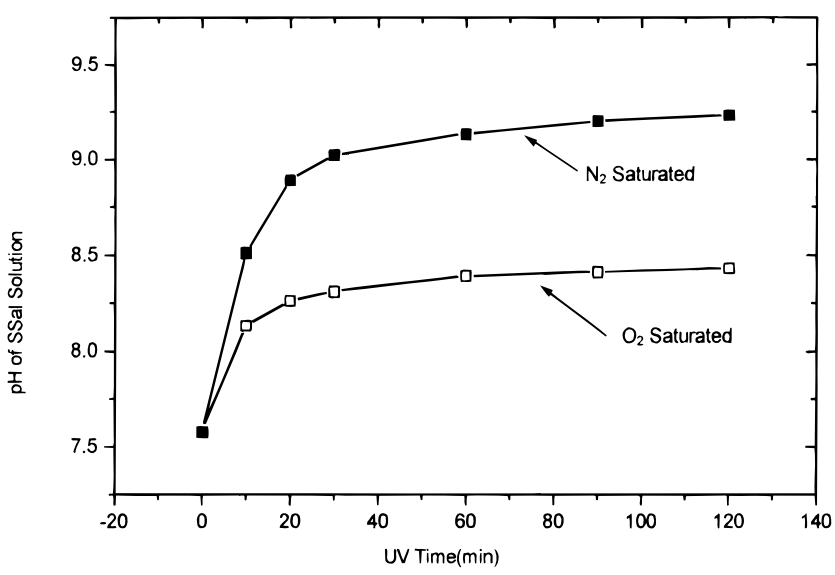

Figure 5. $\mathrm{pH}$ change during SSal photoelectrochemical degradation: bias $=700 \mathrm{mV}$, gas flow $=1000 \mathrm{~mL} \mathrm{~min}^{-1}$, SSal concentration $=$ $5.12 \times 10^{-4} \mathrm{~mol} \mathrm{~L}^{-1}$.

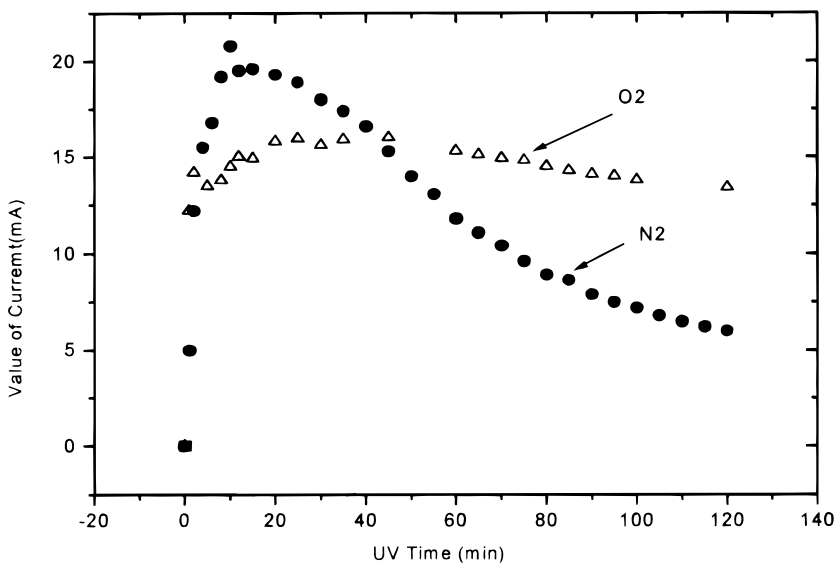

Figure 6. Current change during SSal photoelectrochemical degradation: SSal concentration $=1.97 \times 10^{-4} \mathrm{~mol} \mathrm{~L}^{-1}$, bias $=0.7 \mathrm{~V}(\mathrm{vs}$

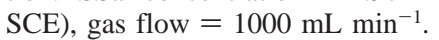

acceptor when $\mathrm{O}_{2}$ is absent. Due to $\mathrm{H}^{+}$involvement, the $\mathrm{pH}$ value changes more drastically in the presence of $\mathrm{N}_{2}$ than in the presence of $\mathrm{O}_{2}$. Whereas, when $\mathrm{O}_{2}$ acts as the electron acceptor, the decrease of the SSal amount is mainly attributed to the $\mathrm{pH}$ change. On the other hand, $\mathrm{H}^{+}$is moving to the counter electrode and therefore causes the external current to change more obviously, while when $\mathrm{O}_{2}$ was continuously supplied, it is unable to change the external current so remarkably.

EIS Characteristics of SSal Photoelectrochemical Degradation. It is found that for the SSal photoelectrochemical degradation, only one arc and only one peak can be observed on the EIS Nynquist plot (Figure 7) and on the EIS Bode plot (Figure 8), respectively, suggesting that such a degradation reaction appears to be a simple electrode reaction and the electrode potential is the controlling parameter. This result is in agreement with the concept that the photoelectrochemical degradation reaction was caused by the photogenerated electronhole pairs which are separated by the external bias. Provided the proper light source is selected, the electrode potential is the controlling parameter of the speed of the electrode kinetic reaction.

Since only electrode potential is acting as the controlling parameter, the impedance of the electrode is:

$$
Z=R_{\mathrm{s}}+\frac{1}{R_{\mathrm{t}}+j \omega C_{\mathrm{dl}}}
$$




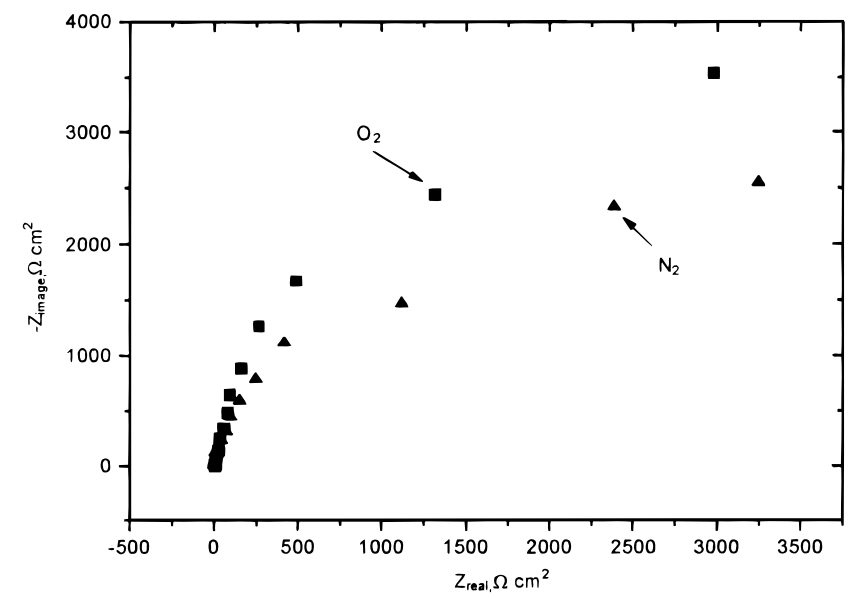

Figure 7. EIS Nyquist plots of SSal photoelectrochemical degradation: SSal concentration $=5.0 \times 10^{-4} \mathrm{~mol} \mathrm{~L}^{-1}$, external bias $=$ $50 \mathrm{mV}$.

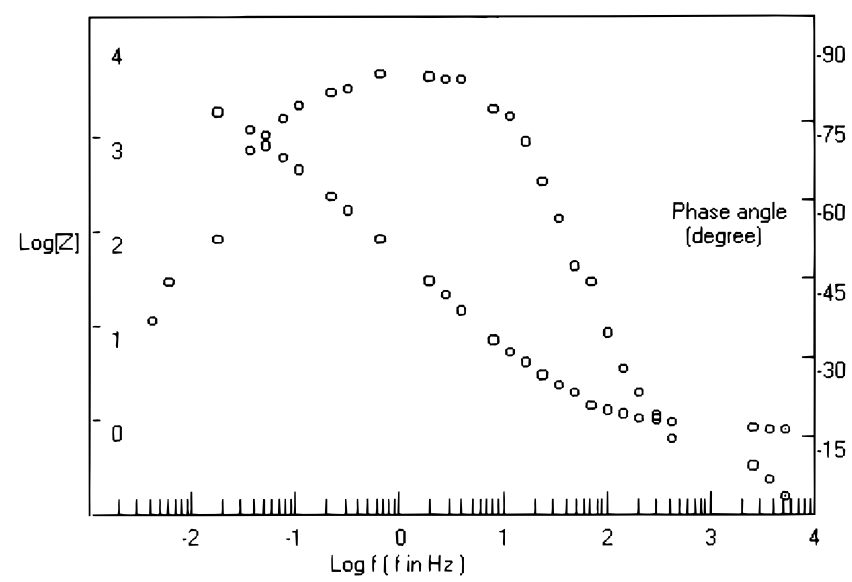

Figure 8. EIS Bode plots of SSal photoelectrochemical degradation $\mathrm{N}_{2}$-purged, other experimental conditions are the same as in Figure 7.

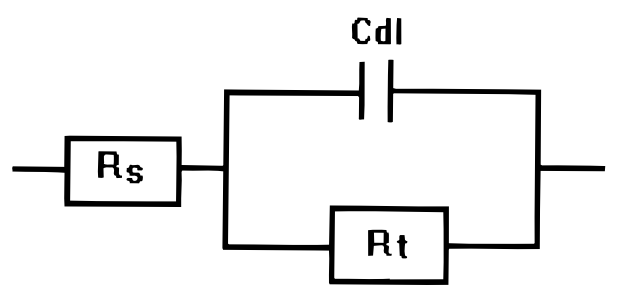

Figure 9. The equivalent circuit of the photoelectrochemical degradation of SSal.

where $Z$ is the electrode impedance, $R_{\mathrm{s}}$ is the solution resistance, $R_{\mathrm{t}}$ is the electron-transfer resistance, $\omega$ is the angle frequency, and $C_{\mathrm{dl}}$ is the capacitance of double layer, $j=(-1)^{1 / 2}$. Accordingly, the equivalent circuit of the photoelectrochemical degradation of SSal is the parallel connection of $C_{\mathrm{dl}}$ and $R_{\mathrm{t}}$ and then the serial connection with $R_{\mathrm{s}}$ (Figure 9).

Reaction Rates and EIS Characteristics. The size of the arc radius on the EIS Nynquist plot means the rate of electrode reaction if there is only one arc to be observed. Figure 10 shows that the combination of the bias potential and the UV illumination causes the smallest arc radius on the EIS Nyquist plot, meaning an effective separation of photogenerated electronhole pairs and the effective photoelectrocatalytic degradation of SSal. Meanwhile, the size of the arc radius can be shown by the value of electron-transfer resistance $\left(R_{\mathrm{t}}\right)$ which indicates the rate of the electrochemical reaction. Table 2 gives such resistance values and the first-order constants of the photoelectrochemical degradations. Note that the values of $R_{\mathrm{t}}$ were

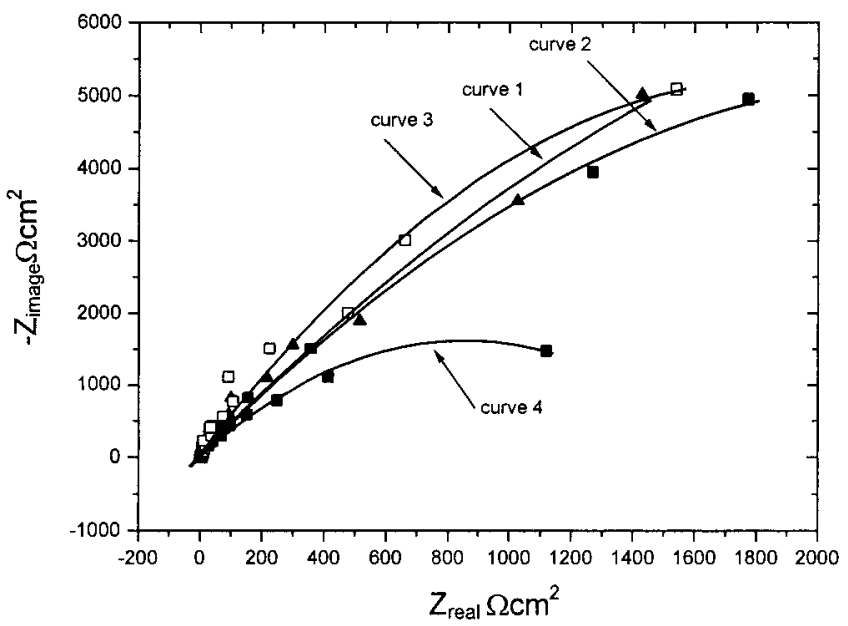

Figure 10. EIS Nynquist plots of SSal photoelectrochemical degradations: SSal concentration $=5.0 \times 10^{-4} \mathrm{~mol} \mathrm{~L}^{-1}$. Curve 1, dark, no bias; curve 2 , dark, bias $=50 \mathrm{mV}$; curve 3 , illumination, no bias; curve 4 , illumination, bias $=50 \mathrm{mV}$.

TABLE 2: Values of Electron Transfer Resistance $\left(R_{t}\right)$ and First-Order Constants of the SSal Degradation Reactions $(k)$

\begin{tabular}{cccl}
\hline number & $\begin{array}{c}R_{\mathrm{t}} \\
(\mathrm{ohms})\end{array}$ & $\begin{array}{c}k \\
\left(\mathrm{~min}^{-1}\right)\end{array}$ & \multicolumn{1}{c}{ impedance experimental conditions } \\
\hline $\mathrm{A}_{1}$ & $9.67 \times 10^{2}$ & 0.0153 & $\mathrm{~N}_{2}$-purged, illumination, bias $=50 \mathrm{mV}$ \\
$\mathrm{A}_{2}$ & $1.11 \times 10^{3}$ & 0.0169 & $\mathrm{O}_{2}$-purged, illumination, bias $=50 \mathrm{mV}$ \\
$\mathrm{A}_{3}$ & $1.03 \times 10^{4}$ & 0.0087 & $\mathrm{O}_{2}$-purged, illumination, no bias \\
$\mathrm{A}_{4}$ & $2.61 \times 10^{4}$ & 0.0008 & $\mathrm{~N}_{2}$-purged, dark, bias = 50 $\mathrm{mV}$ \\
$\mathrm{A}_{5}$ & $2.74 \times 10^{4}$ & 0.0008 & $\mathrm{~N}_{2}$-purged, illumination, no bias \\
$\mathrm{A}_{6}$ & $2.82 \times 10^{4}$ & 0 & $\mathrm{~N}_{2}$-purged, dark, no bias
\end{tabular}

simulated from eq 1 and the equivalent (Figure 9), both of which correspond to the EIS plots (see Figure 10) using the software written by ourselves, ${ }^{22}$ and that the values of $k$ were obtained from the kinetic experiments of the photoelectrochemical degradation reactions.

It is worth noting that the arc radius for the $\mathrm{N}_{2}$-purged condition is smaller than that for the $\mathrm{O}_{2}$-purged condition (see Figure 4), while the first-order constant of the $\mathrm{O}_{2}$ condition is larger that that of the $\mathrm{N}_{2}$ condition (see Table 2). It may be explained that, in the presence of $\mathrm{O}_{2}$, SSal can be degraded photochemically in some degree even in the absence of bias potential and $\mathrm{TiO}_{2}$, causing the value of the first- order constant to be apparently larger than that under the $\mathrm{N}_{2}$-purged condition. However, the part of SSal photochemical degradation cannot be detected by EIS because it takes place in the solution phase instead of on the electrode surface. In the meaning of electrode surface reaction, the SSal photoelectrochemical degradation under the $\mathrm{N}_{2}$-purged condition is more rapid than that under the $\mathrm{O}_{2}$-purged condition (see the arc radius in Figure 10). Therefore, it can be concluded that the values of $R_{\mathrm{t}}$ reflect the rates of SSal degradation reactions.

Separation of Photogenerated Electron-Hole Pairs. A necessary step for semiconductor photocatalytic degradation is the generation and separation of electron-hole pairs. In the three-electrode system with photocatalyst as the anode, when the semiconductor is illuminated, its Fermi band is elevated, and the Fermi band of the counter electrode is elevated at the same time because it is linked with the semiconductor anode. Possibly, when the Fermi band of the counter electrode becomes higher than that of the red/ox couple, the electrons escape from the semiconductor surface to the counter electrode and an external current is expected even if there is no bias potential. If the Fermi band of the counter electrode is still lower than that of the red/ox couple, an anodic bias potential is expected to 


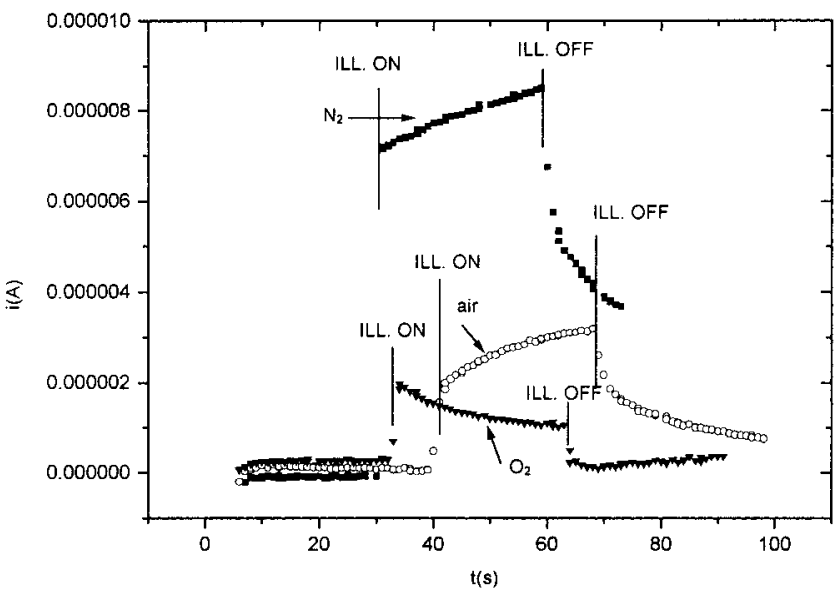

Figure 11. Photo current response of the three-electrode system in SSal medium: $\mathrm{pH}=7.5$, SSal concentration $=1.8 \times 10^{-5} \mathrm{~mol} \mathrm{~L}^{-1}$.

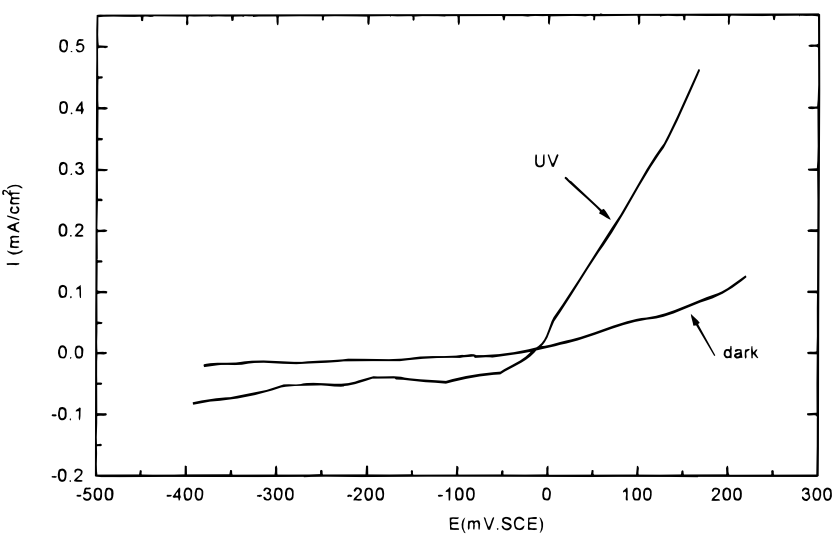

Figure 12. The $i-V$ characteristics of $\mathrm{TiO}_{2}$ on porous nickel reference electrode $=\mathrm{SCE}$, scan rate $=25 \mathrm{mV} \mathrm{s}^{-1}$, SSal concentration $=5.0 \times$ $10^{-4} \mathrm{~mol} \mathrm{~L}^{-1}$.

elevate it. Figure 11 shows that when the three-electrode system is short-circuited, photocurrent response was detected upon illumination even without external potential, indicating that the photogenerated electrons are moving through the external circuit. Figure 11 also shows that the value of photo current response becomes larger with the decrease of the amount of oxygen in the system. When oxygen is present around the anode, some photogenerated electrons transfer directly to $\mathrm{O}_{2}$ on the anode surface and fail to arrive at the counter electrode, thus the determined current value decreases.

In this work, effective degradation of SSal was not observed without an anodic bias potential, although the photo response current was detected. The anode and the cathode behave as a capacitor. Therefore, the immobilized semiconductor photocatalyst is attached to a conductive support on which a bias potential is imposed to result in better electron-hole separation and more effective SSal degradation (see Figure 10 and Table 2). Figure 12 shows that when anodic bias potential is present, an obvious anodic photo current is obtained, and that illumination with a $365 \mathrm{~nm}$ lamp contributes a lot to the current in external current due to the flux of photogenerated electrons.

\section{Conclusion}

Sulfosalicylic acid could be photoelectrochemically degraded effectively as the external potential was up to $700 \mathrm{mV}$ (vs SCE). When the externally applied potential was $600 \mathrm{mV}$ (vs SCE), such degradation appeared to take place much more slowly. It is shown by the EIS that the photoelectrocatalytic degradation appears to be a simple reaction on the electrode surface, suggesting that only one step of charge transfer is involved in the electrode process. The value of the resistance of charge transfer for the photoelectrocatalytic reaction of SSal not only indicates the reaction rate, but also indicates the separation efficiency of the electron-hole pairs. The separation efficiency of the electron-hole pairs under $\mathrm{N}_{2}$ atmosphere is higher than that under $\mathrm{O}_{2}$ atmosphere. Studies on the possibility that EIS can be employed to predict the rates of organic degradations under different conditions or to evaluate the catalytic activity of different photocatalysts is under way in our laboratory.

Acknowledgment. Support from the National Natural Science Foundation of China (No. 29877024), the Chinese Academy of Sciences, and the China Postdoctoral Science Foundation is acknowledged. The authors thank Doctor Anbao Yuan and Doctor Longjun Leng for their help in the EIS measurements.

\section{References and Notes}

(1) Ollis, D. F. Environ. Sci. Technol. 1985, 19, 480

(2) Hoffmann, M. R.; Martin, S. T.; Choi, W., et al. Chem. Rev. 1995, $95,69$.

(3) Kormann, C.; Bahnemann, D. W.; Hoffmann, M. R. Environ. Sci. Technol. 1991, 25, 494.

(4) Bianco, A.; Vincenti, M.; Bianciotto, A., et al. Appl. Catal., B. Environmental 1999, 22, 149.

(5) Byrne, J. A.; Eggins, B. R.; Brown, N. M. D., et al. Appl. Catal., B: Environmental 1998, 17, 25.

(6) Vinodgopal, V.; Hotchandani, S.; Kamat, P. V. J. Phys. Chem. 1993 , 97, 9040 .

(7) Kim, H.; Anderson, C. Environ. Sci. Technol. 1994, 28, 479.

(8) Vinodgopal, K.; Kamat, P. V. Environ. Sci. Technol. 1995, 29, 841.

(9) Kesselman, J. M.; Lewis, N. S.; Hoffmann, M. R. Environ. Sci. Technol. 1997, 31, 2298.

(10) Tacconi, N. R. D.; Wenren, H.; McCheseney, D., et al. Langmuir 1998, 14, 2933.

(11) Fujishima, A.; Honda, K. Nature 1972, 238, 37.

(12) Rajeshwar, K. J. Appl. Chem. 1995, 25, 1067.

(13) Chiang, C.; Chang, J. E. Water Res. 1995, 29, 671.

(14) Pelegrini, R.; Peralta-Zamora, P.; Andrade, A. R. de, et al. Appl. Catal., B: Environmental 1999, 22, 83.

(15) Kesselman, J. M.; Shreve, G. A.; Hoffmann, M. R., et al. J. Phys. Chem. 1994, 98, 13385.

(16) Gomes, W. P.; Vanmaekelbergh, D. Electrochim. Acta 1996, 41, 967.

(17) Hens, Z. J. Phys. Chem. B 1999, 103, 122.

(18) Liu, H.; Cheng, S. A.; Zhang, J. Q., et al. Chemosphere 1999, 38, 283.

(19) Zhang, F.; Zhao, J.; Shen, T., et al. Appl. Catal., B: Environmental 1998, 122, 178. 95.

(20) Hidaka, H.; Ajisaka, K.; Horikoshi, S., et al. Catal. Lett. 1999, 60,

(21) Vinodgopal, K.; Stafford, U.; Gray, K. A., et al. J. Phys. Chem. 1994, 98, 6797.

(22) Cheng, S. A.; Zhang, J. Q.; Liu, H., et al. J. Power Sources 1998, 74,155 . 